\title{
SUBCLASSES OF UNIVALENT FUNCTIONS SUBORDINATE TO CONVEX FUNCTIONS
}

\section{YONG CHAN KIM}

Department of Mathematics

Yeungnam University

Gyongsan 712-749, KOREA

\section{IL BONG JUNG}

Department of Mathematics

Kyungpook National University Taegu 702-701, KOREA

(Received May 17, 1995 and in revised form August 30, 1995)

ABSTRACT. In this paper, we define a new subclass $\mathcal{M}_{\alpha}(A, B)$ of univalent functions and investigate several interesting characterization theorems involving a general class $\mathcal{S}^{*}[A, B]$ of starlike functions

KEY WORDS AND PHRASES: Univalent function, subordination, $\alpha$-convex function 1991 AMS SUBJECT CLASSIFICATION CODES: 30C45, 30D30

\section{INTRODUCTION AND DEFINITIONS}

Let $\mathcal{A}$ denote the class of functions normalized by

$$
f(z)=z+\sum_{n=2}^{\infty} a_{n} z^{n}
$$

which are analytic in the open unit disk $\mathcal{U}=\{z: z \in \mathbb{C}$ and $|z|<1\}$. Further, let $\mathcal{S}$ denote the class of all functions in $\mathcal{U}$ which are univalent in $\mathcal{U}$

A function $f(z)$ belonging to $\mathcal{S}$ is said to be starlike of order $\alpha(0 \leq \alpha<1)$ if and only if

$$
\operatorname{Re}\left(\frac{z f^{\prime}(z)}{f(z)}\right)>\alpha \quad(z \in \mathcal{U} ; 0 \leq \alpha<1) .
$$

We denote by $\mathcal{S}^{*}(\alpha)$ the subclass of $\mathcal{S}$ consisting of functions which are starlike of order $\alpha$

A function $f(z)$ belonging to $\mathcal{S}$ is said to be convex of order $\alpha(0 \leq \alpha<1)$ if and only if

$$
\operatorname{Re}\left(1+\frac{z f^{\prime \prime}(z)}{f^{\prime}(z)}\right)>\alpha \quad(z \in \mathcal{U} ; 0 \leq \alpha<1) .
$$

We denote by $\mathcal{K}(\alpha)$ the subclass of $\mathcal{S}$ consisting of functions which are convex of order $\alpha$ We note that

$$
\mathcal{S}^{*}(\alpha) \subseteq \mathcal{S}^{*}(0) \equiv \mathcal{S}^{*} \quad(0 \leq \alpha<1)
$$

and

$$
\mathcal{K}(\alpha) \subseteq \mathcal{K}(0) \equiv \mathcal{K} \quad(0 \leq \alpha<1) .
$$

With a view to introducing an interesting family of analytic functions, we should recall the concept of subordination between analytic functions Given two functions $f(z)$ and $g(z)$, which are analytic in $\mathcal{U}$, the function $f(z)$ is said to be subordinate to $g(z)$ if there exists a function $h(z)$, analytic in $\mathcal{U}$ with

$$
h(0)=0 \text { and }|h(z)|<1 \text {, }
$$

such that

$$
f(z)=g(h(z)) \quad(z \in \mathcal{U}) .
$$

We denote this subordination by 


$$
f(z) \prec g(z) .
$$

In particular, if $g(z)$ is univalent in $\mathcal{U}$, the subordination $(18)$ is equivalent to

$$
f(0)=g(0) \text { and } f(\mathcal{U}) \subset g(\mathcal{U}) .
$$

Janowski [1] introduced the class $\mathcal{P}[A, B]$ For $-1 \leq B<A \leq 1$, a function $p$, analytic in $\mathcal{U}$ with $p(0)=1$, belongs to the class $\mathcal{P}[A, B]$ if $p(z)$ is subordinate to $(1+A z) /(1+B z)$ Also $\mathcal{S}^{*}[A, B]$ and $\mathcal{K}[A, B]$ denote the subclasses of $\mathcal{S}$ consisting of all functions $f(z)$ such that

$$
\frac{z f^{\prime}(z)}{f(z)} \in \mathcal{P}[A, B] \text { and } \frac{\left(z f^{\prime}(z)\right)^{\prime}}{f^{\prime}(z)} \in \mathcal{P}[A, B]
$$

respectively. We note that $\mathcal{S}^{*}[-1,1]=\mathcal{S}^{*}$ and $\mathcal{K}[-1,1]=\mathcal{K}$

DEFINITION 1. Let $\alpha$ be a real number. A function $f(z)$ belonging to the class $\mathcal{A}$ with $(f(z) / z) f^{\prime}(z) \neq 0$ is said to be $\alpha$-convex in $\mathcal{U}$ if and only if

$$
\operatorname{Re}\left[(1-\alpha) \frac{z f^{\prime}(z)}{f(z)}+\alpha\left(1+\frac{z f^{\prime \prime}(z)}{f^{\prime}(z)}\right)\right]>0 .
$$

Also we denote the class of $\alpha$-convex functions by $\mathcal{M}_{\alpha}$. Then it is easy to see that

$$
\mathcal{M}_{\alpha}=\left\{f \in \mathcal{S}: \operatorname{Re}\left[(1-\alpha) \frac{z f^{\prime}(z)}{f(z)}+\alpha\left(1+\frac{z f^{\prime \prime}(z)}{f^{\prime}(z)}\right)\right]>0, \quad z \in \mathcal{U}\right\} .
$$

See Eenigenberg and Miller [5] for further information on them

We now define the class $\mathcal{M}_{\alpha}(A, B)$ as follows: If $\alpha$ is a real number, then

$$
\begin{array}{r}
\mathcal{M}_{\alpha}(A, B)=\left\{f \in \mathcal{S}:\left[(1-\alpha) \frac{z f^{\prime}(z)}{f(z)}+\alpha\left(1+\frac{z f^{\prime \prime}(z)}{f^{\prime}(z)}\right)\right] \prec \frac{1+A z}{1+B z},\right. \\
-1 \leq B<A \leq 1, z \in \mathcal{U}\} .
\end{array}
$$

Clearly, we have

$$
\mathcal{M}_{\alpha}(1,-1)=\mathcal{M}_{\alpha}, \quad M_{1}(A, B)=\mathcal{K}[A, B]
$$

and

$$
\mathcal{M}_{0}(A, B)=\mathcal{S}^{*}[A, B]
$$

\section{MAIN RESULTS}

Applying the method of the integral representation [2] for functions in $\mathcal{M}_{\alpha}(A, B)(\alpha>0)$, it is not difficult to deduce

LEMMA 1. The function $f(z)$ is in $\mathcal{M}_{\alpha}(A, B), \alpha>0$, if and only if there exists a function $g(z)$ belonging to the class $\mathcal{S}^{*}[A, B]$ such that

$$
f(z)=\left[\frac{1}{\alpha} \int_{0}^{z}\{g(t)\}^{1 / \alpha} t^{-1} d t\right]^{\alpha} .
$$

PROOF. Setting $g(z)=f(z)\left\{z f^{\prime}(z) / f(z)\right\}^{\alpha}$, so that (2.1) is satisfied, we observe that

$$
\frac{z g^{\prime}(z)}{g(z)}=(1-\alpha) \frac{z f^{\prime}(z)}{f(z)}+\alpha\left(1+\frac{z f^{\prime \prime}(z)}{f^{\prime}(z)}\right) \text {. }
$$

Hence $f \in \mathcal{M}_{\alpha}(A, B)$ if and only if $g \in \mathcal{S}^{*}[A, B]$.

Before stating our first theorem, we need the following definition

DEFINITION 2. Let $c$ be a complex number such that $\operatorname{Re} c>0$, and let 


$$
N=N(c)=\left[|c|(1+2 \operatorname{Re} c)^{1 / 2}+\operatorname{Im} c\right] / \operatorname{Re} c .
$$

If $h$ is the univalent function $h(z)=2 N z /\left(1-z^{2}\right)$ and $b=h^{-1}(c)$, then we define the "open door" (cf [3]) function $\mathcal{Q}_{c}$ as

$$
\mathcal{Q}_{c}(z)=h[(z+b) /(1+\bar{b} z)], \quad z \in \mathcal{U} .
$$

THEOREM 1. Let $f \in \mathcal{M}_{\alpha}(A, B)(\alpha>0)$, and let

$$
\left(\frac{1+A z}{1+b z}\right) \prec \alpha \mathcal{Q}_{\frac{1}{\alpha}}(z)
$$

Then $f \in \mathcal{S}^{*}$

PROOF. Since $f \in \mathcal{M}_{\alpha}(A, B)(\alpha>0)$, it follows that there exists a function $g \in \mathcal{S}^{*}[A, B]$ such that

$$
f(z)=\left[\frac{1}{\alpha} \int_{0}^{z}\{g(t)\}^{1 / \alpha} t^{-1} d t\right]^{\alpha}
$$

by using Lemma 1. By the hypothesis, we also have

$$
\frac{1}{\alpha}\left(\frac{z g^{\prime}(z)}{g(z)}\right) \prec \frac{1}{\alpha}\left(\frac{1+A z}{1+B z}\right) \prec \mathcal{Q}_{\frac{1}{\alpha}}(z) .
$$

Thus, by a result of Miller and Mocanu ([3], Corollary 3.1), we have

$$
f(z)=\left[\frac{1}{\alpha} \int_{0}^{z}\{g(t)\}^{1 / \alpha} t^{-1} d t\right]^{\alpha} \in \mathcal{S}^{*}
$$

LEMMA 2. (Mocanu [4]) Let $\mathcal{P}$ be an analytic function in $\mathcal{U}$ satisfying $\mathcal{P} \prec \mathcal{Q}_{c}$ If $p$ is analytic in $\mathcal{U}, p(0)=1 / c$, and

$$
z p^{\prime}(z)+\mathcal{P}(z) p(z)=1
$$

then $\operatorname{Re} p(z)>0$ in $\mathcal{U}$

Making use of Lemma 2, we now prove

THEOREM 2. Let $f \in \mathcal{M}_{\alpha}(A, B)(\alpha>0)$, and let

$$
\frac{z f^{\prime}(z)}{f(z)}+\frac{f(z)}{z f^{\prime}(z)}-1 \prec \mathcal{Q}_{1} \text {. }
$$

Then $f \in \mathcal{S}^{*}[A, B]$.

PROOF. If we set $p(z)=z f^{\prime}(z) / f(z)$, then

$$
p(z)+\frac{z p^{\prime}(z)}{p(z)}=1+\frac{z f^{\prime \prime}(z)}{f^{\prime}(z)} .
$$

Hence

$$
(1-\alpha) \frac{z f^{\prime}(z)}{f(z)}+\alpha\left(1+\frac{z f^{\prime \prime}(z)}{f^{\prime}(z)}\right)=p(z)+\alpha \frac{z p^{\prime}(z)}{p(z)} .
$$

Since $f \in \mathcal{M}_{\alpha}(A, B)$,

$$
p(z)+\alpha \frac{z p^{\prime}(z)}{p(z)} \prec \frac{1+A z}{1+B z}
$$

Setting $\mathcal{P}(z)=p(z)+1 / p(z)-1$, we obtain

$$
z p^{\prime}(z)+\mathcal{P}(z) p(z)=1
$$


and $\mathcal{P} \prec \mathcal{Q}_{1}$ by the hypothesis (2.8)

Thus, by Lemma 2 , we have

$$
\operatorname{Re} p(z)>0 \quad(z \in \mathcal{U})
$$

Since $\alpha>0$,

$$
\operatorname{Re}\left\{\frac{1}{\alpha} p(z)\right\}>0 \quad(z \in \mathcal{U})
$$

Also $(1+A z) /(1+B z)$ (with $-1 \leq B<A \leq 1$ ) is a convex univalent function Therefore, by appealing to a known result ([6], Theorem 7), we conclude from (2 11) and (2 14) that

$$
p(z) \prec \frac{1+A z}{1+B z} .
$$

This evidently completes the proof of Theorem 2

As an example of ([7], Corollary 3.2, see also [9]), consider the case when $\alpha>0$, $-1 \leq B<A \leq 1$, and $A \neq B$. Then the differential equation

$$
q(z)+\alpha \frac{z q^{\prime}(z)}{q(z)}=\frac{1+A z}{1+B z}
$$

has a univalent solution given by

$$
q(z)= \begin{cases}\frac{z^{\frac{1}{\alpha}}(1+B z)^{\frac{1}{\alpha}\left(\frac{A-B}{B}\right)}}{\frac{1}{\alpha} \int_{0}^{z} t^{\frac{1}{\alpha}-1}(1+B t)^{\frac{1}{\alpha}\left(\frac{A-B}{B}\right)} d t} & \text { if } B \neq 0 \\ \frac{z^{\frac{1}{\alpha}} e^{\frac{A}{\alpha} z}}{\frac{1}{\alpha} \int_{0}^{z} t^{\frac{1}{\alpha}-1} e^{\frac{1}{\alpha}} d t} & \text { if } B=0 .\end{cases}
$$

If $p(z)$ is analytic in $\mathcal{U}$ and satisfies

$$
p(z)+\alpha \frac{z p^{\prime}(z)}{p(z)} \prec \frac{1+A z}{1+B z}
$$

then

$$
p(z) \prec q(z) \prec \frac{1+A z}{1+B z} .
$$

Hence, by the equations (2.11) and (2 19), we obtain

THEOREM 3. Let $\alpha>0$ and $f \in \mathcal{M}_{\alpha}(A, B)$. Then

$$
\frac{z f^{\prime}(z)}{f(z)} \prec q(z) \prec \frac{1+A z}{1+B z},
$$

where $q(z)$ is given by (2.17).

THEOREM 4. $\mathcal{K}(\alpha) \subset \mathcal{M}_{\alpha}(1-2 \alpha,-1)(0 \leq \alpha<1)$.

PROOF. If we define

$$
h_{\alpha}(z)=\frac{1+(1-2 \alpha) z}{1-z} \quad(0 \leq \alpha<1),
$$

then we can easily see that $f \in \mathcal{K}(\alpha)$ if and only if

$$
1+\frac{z f^{\prime \prime}(z)}{f^{\prime}(z)} \prec h_{\alpha}(z)
$$

(cf [10], Equation (9)). Hence, by Theorem 1 of [10], we have 


$$
\frac{z f^{\prime}(z)}{f(z)} \prec h_{\alpha}(z)
$$

Therefore we conclude from [8, Lemma 2.2] that

$$
\left[(1-\alpha) \frac{z f^{\prime}(z)}{f(z)}+\alpha\left(1+\frac{z f^{\prime \prime}(z)}{f^{\prime}(z)}\right)\right] \prec h_{\alpha}(z) .
$$

This completes the proof of Theorem 4

ACKNOWLEDGMENT. This work was partially supported by KOSEF (project No 94-1400-02-01-3) and TGRC-KOSEF, and by the Basic Science Research Institute Program (BSRI-95-1401)

\section{REFERENCES}

[1] JANOWSKI, W, Some extremal problems for certain families of analytic functions I, Ann. Polon. Math. 28 (1973), 297-326

[2] MOCANU, P T., Une propriété de convexité généraliseé dans la théorie de la représentation conforme, Mathematica (Cluj) 11 (34) (1969), 127-133.

[3] MILLER, S.S. and MOCANU, P.T., Classes of univalent integral operators, J. Math. Anal. Appl. 157 (1991), 147-165

[4] MOCANU, P.T., Some integral operators and starlike functions, Rev. Roumaine Math. Pures Appl. 31 (1986), 231-235.

[5] EENIGENBURG, P.J. and MILLER, S.S., The $H^{p}$ classes for $\alpha$-convex functions, Proc. Amer. Math. Soc. 38 (1973), 558-562.

[6] MILLER, S.S and MOCANU, P.T., Differential subordinations and univalent functions, Michigan Math. J. 28 (1981), 157-171

[7] MILLER, S.S. and MOCANU, P T., Univalent solutions of Briot-Bouquet differential equations, J. Differential Equation 56 (1985), 297-309.

[8] NOOR, K.I., On some univalent integral operators, J. Math. Anal. Appl. 128 (1987), 586-592

[9] OWA, S. and SRIVASTAVA, H.M., Analytic solutions of a class of Briot-Bouquet differential equations, in Current Topics in Analytic Function Theory (H.M Srivastava and S Owa, Editors), 252-259, World Science Publishing Company, Singapore, New Jersey, London, and Hong Kong, 1992.

[10] SALAGEAN, G S., Subclasses of univalent functions, Complete Analysis: Fifth Romanıan-Finish Seminer, Part 1 (C Andreian Cazacu, N. Boboc, M Jurchescu, and I Siciu, Editors), 362-372, Springer-Verlag, Berlin, Heidelberg, New York, and Tokyo, 1983 


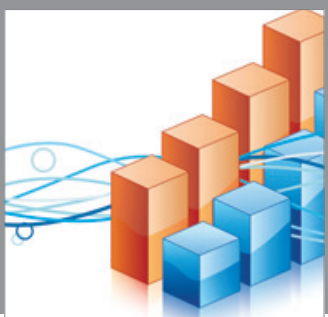

Advances in

Operations Research

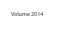

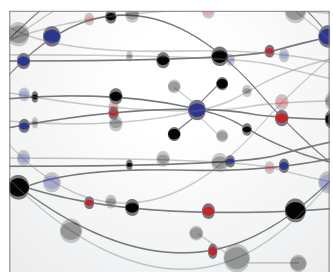

\section{The Scientific} World Journal
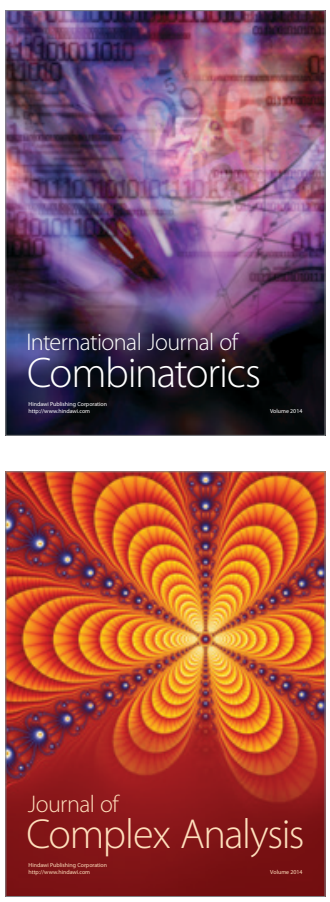

International Journal of

Mathematics and

Mathematical

Sciences
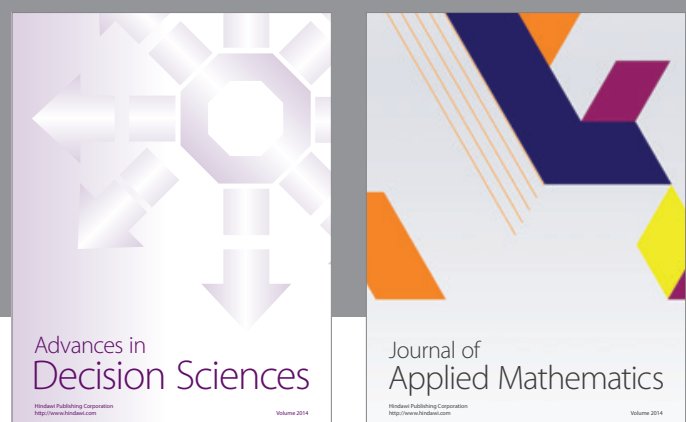

Journal of

Applied Mathematics
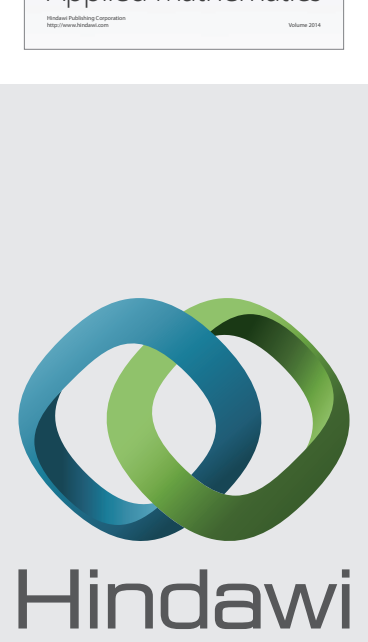

Submit your manuscripts at http://www.hindawi.com
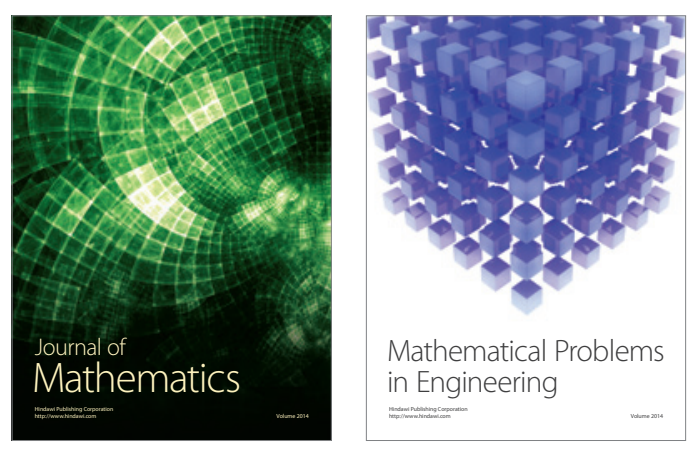

Mathematical Problems in Engineering
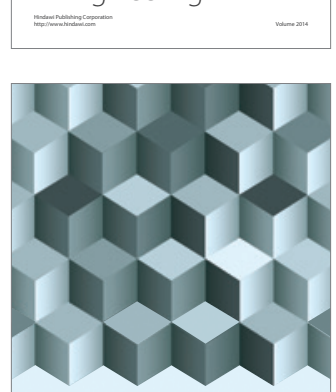

Journal of

Function Spaces
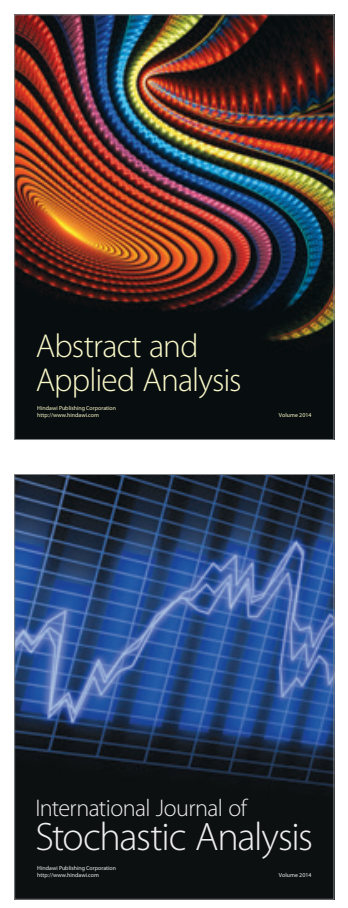

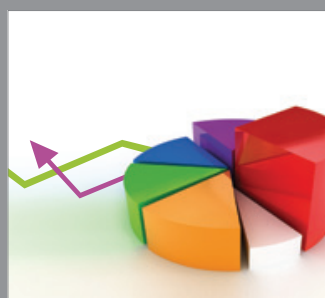

ournal of

Probability and Statistics

Promensencen
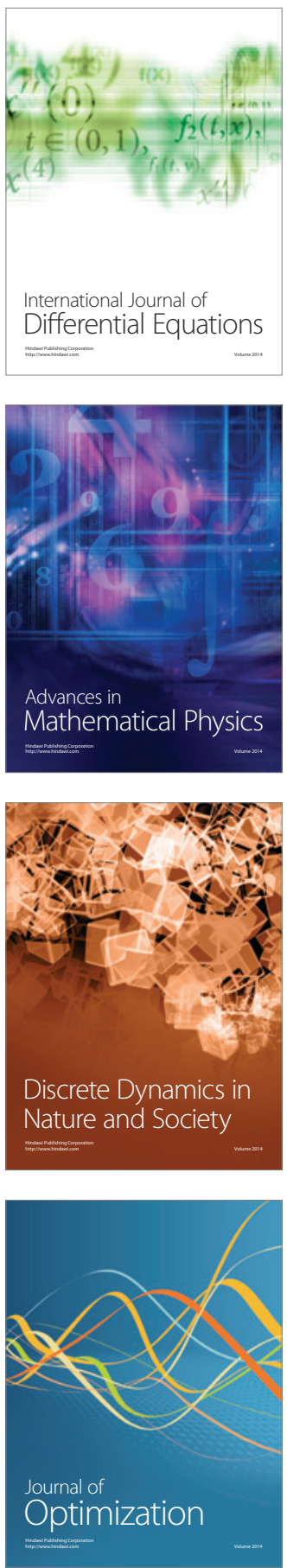